\title{
Expression of tumor suppressor REIC/Dkk-3 by a newly improved adenovirus vector with insertion of a hTERT promoter at the 3 '-side of the transgene
}

\author{
ENDY WIDYA PUTRANTO ${ }^{1,2^{*}}$, RIE KINOSHITA $^{1 *}$, MASAMI WATANABE $^{3,4}$, TAKUYA SADAHIRA $^{3}$, \\ HITOSHI MURATA $^{1}$, KEN-ICHI YAMAMOTO ${ }^{1}$, JUNICHIRO FUTAMI ${ }^{5}$, KEN KATAOKA ${ }^{6}$, \\ YUSUKE INOUE $^{7}$, I. MADE WINARSA RUMA ${ }^{1,8}$, I. WAYAN SUMARDIKA ${ }^{1,8}$, CHEN YOUYI $^{1}$, \\ MIYOKO KUBO $^{1}$, YOSHIHIKO SAKAGUCHI ${ }^{9}$, KENJI SAITO $^{1,10}$, YASUTOMO NASU ${ }^{3}$, \\ HIROMI KUMON $^{10,11}$, NAM-HO HUH ${ }^{1}$ and MASAKIYO SAKAGUCHI ${ }^{1}$
}

\begin{abstract}
${ }^{1}$ Department of Cell Biology, Okayama University Graduate School of Medicine, Dentistry and Pharmaceutical Sciences, Okayama 700-8558, Japan; ${ }^{2}$ Department of Pediatrics, Dr Sardjito Hospital/Faculty of Medicine, Gadjah Mada University, Yogyakarta 55281, Indonesia; ${ }^{3}$ Department of Urology, Okayama University Graduate School of Medicine, Dentistry and Pharmaceutical Sciences; ${ }^{4}$ Center for Innovative Clinical Medicine, Okayama University Hospital, Okayama 700-8558; ${ }^{5}$ Department of Medical and Bioengineering Science, Okayama University Graduate School of Natural Science and Technology, Okayama 700-8530; ${ }^{6}$ Department of Life Science, Faculty of Science,

Okayama University of Science, Okayama 700-0005; ${ }^{7}$ Faculty of Science and Technology, Division of Molecular Science, Gunma University, Kiryu, Gunma 376-8515, Japan; ${ }^{8}$ Faculty of Medicine, Udayana University, Denpasar, Bali 80232, Indonesia; ${ }^{9}$ Department of Microbiology, Kitasato University School of Medicine, Sagamihara, Kanagawa 252-0374; ${ }^{10}$ Niimi College, Niimi, Okayama 718-8585; ${ }^{11}$ Innovation Center Okayama for Nanobio-Targeted Therapy, Okayama University Hospital, Okayama 700-8558, Japan
\end{abstract}

Received August 22, 2016; Accepted March 30, 2017

DOI: $10.3892 / \mathrm{ol} .2017 .6201$

\begin{abstract}
Reduced expression in immortalized cells (REIC)/Dickkopf-3 (Dkk-3) overexpression, induced using an adenovirus (Ad)-REIC, has been revealed to have a dramatic therapeutic effect on multiple types of cancer. To achieve an improved therapeutic effect from Ad-REIC on cancer, our group previously developed an enhanced gene expression system, the C-TSC cassette [cytomegalovirus (CMV)-RU5' located upstream (C); another promoter unit composed of triple tandem promoters, human telomerase reverse transcriptase (hTERT), simian virus 40 and CMV, located downstream of the cDNA (TSC); plus a polyadenylation (polyA) signal]. When applied to the conventional Ad-REIC, this novel system induced the development of an enhanced product, Ad-C-TSC-REIC,
\end{abstract}

Correspondence to: Dr Masakiyo Sakaguchi, Department of Cell Biology, Okayama University Graduate School of Medicine, Dentistry and Pharmaceutical Sciences, 2-5-1 Shikata, Kita, Okayama 700-8558, Japan

E-mail: masa-s@md.okayama-u.ac.jp

${ }^{*}$ Contributed equally

Key words: reduced expression in immortalized cells/Dickkopf-3, gene expression, plasmid, adenovirus, cancer therapy which exhibited a noticeable anticancer effect. However, there were difficulties in terms of Ad-C-TSC-REIC productivity in HEK293 cells, which are a widely used donor cell line for viral production. Productivity of Ad-C-TSC-REIC was significantly reduced compared with the conventional Ad-REIC, as the Ad-C-TSC-REIC had a significantly higher ability to induce apoptotic cell death of not only various types of cancer cell, but also HEK293 cells. The present study aimed to overcome this problem by modifying the C-TSC structure, resulting in an improved candidate: A C-T cassette (C: CMV-RU5' located upstream; T: another promoter unit composed of a single hTERT promoter, located downstream of the cDNA plus a polyA signal), which demonstrated gene expression comparable to that of the C-TSC system. The improved adenovirus REIC/Dkk-3 product with the $\mathrm{C}-\mathrm{T}$ cassette, named Ad-C-T-REIC, exhibited a higher expression level of REIC/Dkk3, similar to that of Ad-C-TSC-REIC. Notably, the vector mitigated the cell death of donor HEK293 cells, resulting in a higher rate of production of its adenovirus. These results indicated that Ad-C-T-REIC has the potential to be a useful tool for application in cancer gene therapy.

\section{Introduction}

The reduced expression in immortalized cells (REIC) gene was isolated using the subtractive hybridization method, as its expression is reduced in a number of human immortalized 
and neoplastic tumor cells (1). Investigation into the sequence homology of REIC using a computerized scientific database revealed that REIC and Dickkopf-3 (Dkk-3) are identical. Dkk-3 belongs to the Dkks gene family $(1,2)$. Therefore, REIC was designated as REIC/Dkk-3. Previous studies by our group have demonstrated that overexpression of REIC/Dkk-3 by an adenovirus (Ad)-REIC vector resulted in a therapeutic effect against numerous human cancer subtypes via a mechanism triggered by endoplasmic reticulum (ER) stress-mediated c-Jun N-terminal kinase (JNK) activation (3-6).

To obtain improved therapeutic effects of Ad-REIC against cancer, our group previously developed a powerful gene expression system; the C-TSC cassette [cytomegalovirus (CMV)-RU5' located upstream (C); another promoter unit composed of triple tandem promoters, human telomerase reverse transcriptase (hTERT), simian virus 40 (SV40) and CMV, located downstream of the cDNA (TSC); plus a polyadenylation (polyA) signal], which exhibited a higher efficacy of REIC/Dkk-3 expression compared with that of conventional vector systems $(7,8)$. Adaptation of the C-TSC cassette and the cargo REIC/Dkk-3 cDNA to an adenovirus vector resulted in Ad-C-TSC-REIC, which induced apoptotic cell death to a greater extent in more types of cancer cells than the usual CMV promoter-driven Ad-REIC (7,8). However, due to the higher efficiency of virus-mediated REIC/Dkk-3 expression levels by Ad-C-TSC-REIC, we have identified that the viral production rate is markedly mitigated in HEK293 cells, which are commonly used in adenovirus expansion as a production donor.

Therefore, the present study aimed to improve the Ad-C-TSC-REIC vector by making it more permissive in donor HEK293 cells, without losing its high expression level in cancer cells. Thus, the C-T cassette was developed by replacing the 3'-TSC tandem promoter unit in the C-TSC cassette with a single hTERT promoter (T). This novel cassette provided comparable expression levels in cancer cells and demonstrated significantly mitigated cell death of donor cells used for adenoviral production, resulting in an increased adenoviral titer. The data obtained in the present study indicated that the improved adenovirus vector carrying REIC/Dkk-3, termed Ad-C-T-REIC, may have the potential to become a more useful tool for its application in cancer gene therapy.

\section{Materials and methods}

Cells. The embryonic kidney cell line HEK293 [American Type Culture Collection (ATCC); Manassas, VA, USA], embryonic kidney cell line HEK293T (ATCC), prostate adenocarcinoma cell line PC-3 (ATCC) and glioblastoma cell line T98 (ATCC) were cultivated in DMEM/F12 medium (Invitrogen; Thermo Fisher Scientific, Inc., Waltham, MA, USA) supplemented with $10 \%$ fetal bovine serum (FBS; Thermo Fisher Scientific, Inc.) at $37^{\circ} \mathrm{C}$ with $5 \% \mathrm{CO}_{2}$. Human umbilical vein endothelial cells (HUVEC; Kurabo Industries Ltd., Osaka, Japan) were grown in Humedia-EG medium (Kurabo Industries Ltd.) supplemented with $2 \% \mathrm{FBS}$, fibroblast growth factor-B $(5 \mathrm{ng} / \mathrm{ml})$, epidermal growth factor $(10 \mathrm{ng} / \mathrm{ml})$, hydrocortisone $(1 \mu \mathrm{g} / \mathrm{ml})$ and heparin $(10 \mu \mathrm{g} / \mathrm{ml})$ at $37^{\circ} \mathrm{C}$ with $5 \% \mathrm{CO}_{2}$.

Preparation of plasmid and adenovirus vectors. The compositions of a series of novel, constructed expression plasmids are presented in Fig. 1A. pIDT-SMART (C-TSC) was previously reported (7). A 189-base pair element of the hTERT promoter (T) [accession no. DQ264729 (1618-1806) from GenBank] was used for modification of pIDT-SMART (C-TSC). Full-length cDNA of human REIC/Dkk-3 was amplified by reverse transcription-polymerase chain reaction and inserted into the indicated vectors.

The improved C-T unit with REIC/Dkk-3 cDNA was cloned into a pShuttle plasmid vector (Takara Biotechnology Co., Ltd., Dalian, China). The resulting donor plasmid was used for constructing an adenovirus vector according to the manufacturer's protocol.

Transfection. Cells were transiently transfected with plasmid vectors using FuGENE-HD (Promega Corporation, Madison, WI, USA) according to the manufacturer's protocol. A negative control used FuGENE-HD alone. Cells were infected with adenovirus vectors at the indicated multiplicity of infection (MOI), as previously described (3-6). Ad-LacZ (Takara Biotechnology Co., Ltd.) was used as a control virus for assessments of apoptosis and to determine infection efficiency.

Western bloting. Cells $\left(1 \times 10^{5}\right.$ cells/well in 6 -well plates) were transiently transfected with plasmid vectors using FuGENE-HD. After $24 \mathrm{~h}$, the transfected cells were collected, treated with M-PER lysis reagent (Invitrogen; Thermo Fisher Scientific, Inc.) and kept on ice for $10 \mathrm{~min}$. Cell debris was then removed by centrifugation at $20,000 \times \mathrm{g}$ at $4^{\circ} \mathrm{C}$ for $20 \mathrm{~min}$. The protein concentration of the supernatant was determined by a Bio-Rad Protein Quantification assay (Bio-rad Laboratories, Inc., Hercules, CA, USA). Samples of the extracted protein $(10 \mu \mathrm{g})$ were separated with $10 \%$ SDS-PAGE. The proteins on the gel were transferred onto a polyvinylidene membrane, which was blocked with $10 \%$ skimmed milk in phosphate-buffered saline with Tween-20 (PBS-T) for $30 \mathrm{~min}$ at room temperature, then incubated with primary antibodies overnight at $4^{\circ} \mathrm{C}$.

The following primary antibodies were used, at a dilution of 1:1,000: Rabbit anti-human REIC/Dkk-3 antibody, which was generated in the Department of Cell Biology, Okayama University Graduate School of Medicine, Dentistry and Pharmaceutical Sciences (Okayama, Japan) (3-6); rabbit anti-human GRP78 [binding immunoglobulin protein (BiP)] antibody (cat. no. ab21685; Abcam, Cambridge, MA, USA); rabbit anti-human stress-activated protein kinases (SAPK)/JNK (cat. no. 9252); mouse anti-human phosphorylated (phosphor)-SAPK/JNK (Thr183/Tyr185) (cat. no. 9255); rabbit anti-human p38 (cat. no. 9212); rabbit anti-human phospho-p38 (Thr180/Tyr182) antibodies (cat. no. 4511; all from Cell Signaling Technology, Inc.) and mouse anti-human tubulin antibody (cat. no. T5168; Sigma-Aldrich; Merck KGaA, Darmstadt, Germany).

The membrane was then washed in PBS-T three times for 10 min and incubated with a secondary antibody diluted to 1:1,000 (anti-rabbit IgG, HRP-linked antibody: cat. no. 7074 or anti-mouse IgG, HRP-linked antibody: cat. no. 7076; Cell Signaling Technology, Inc.) for $1 \mathrm{~h}$ at room temperature. Protein detection was performed with Pierce Western Blotting substrate Plus (Thermo Fisher Scientific, Inc.). All experiments were repeated in triplicate. 
A $\underline{\text { C-TSC cassette }}$
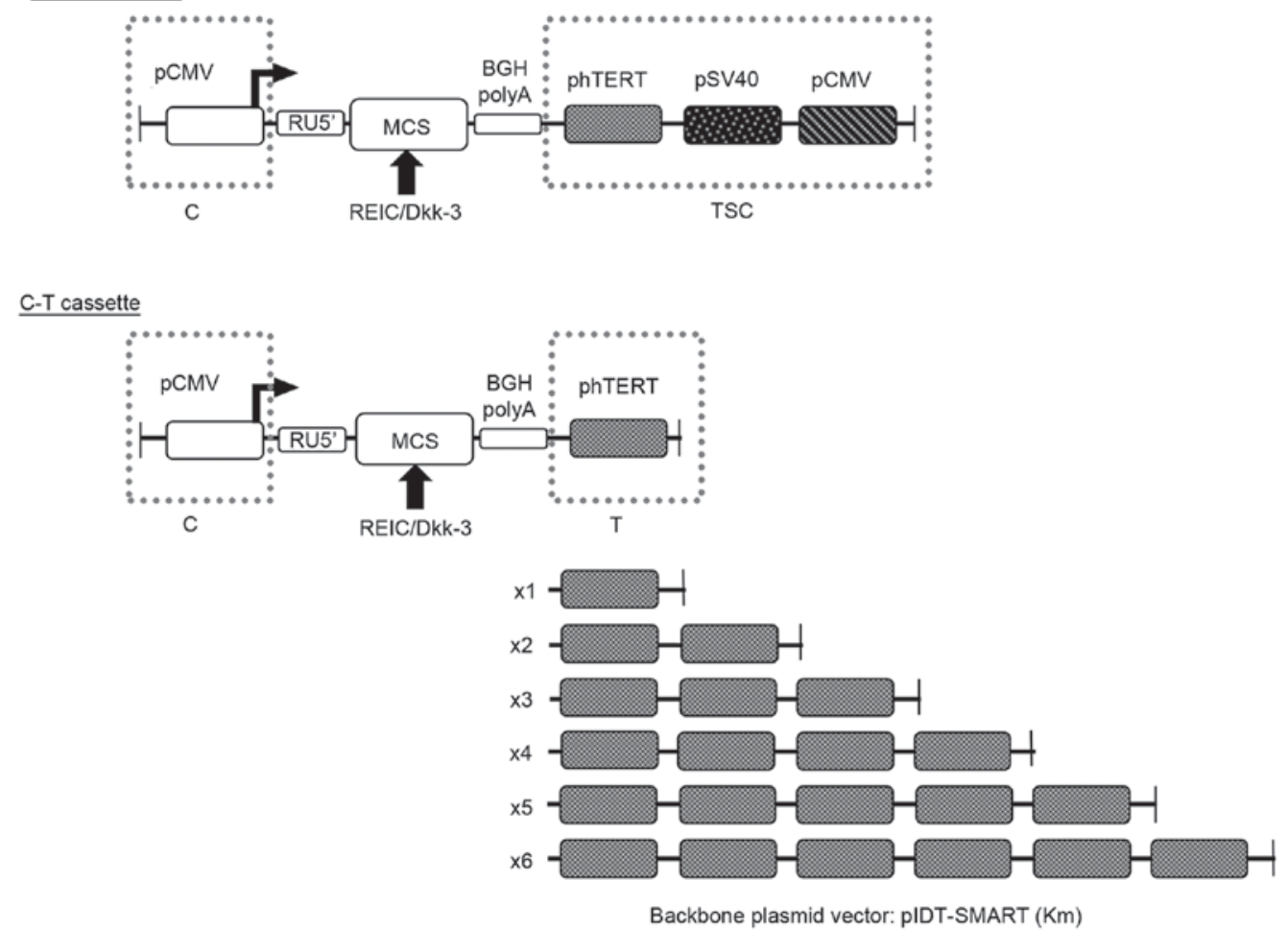

B
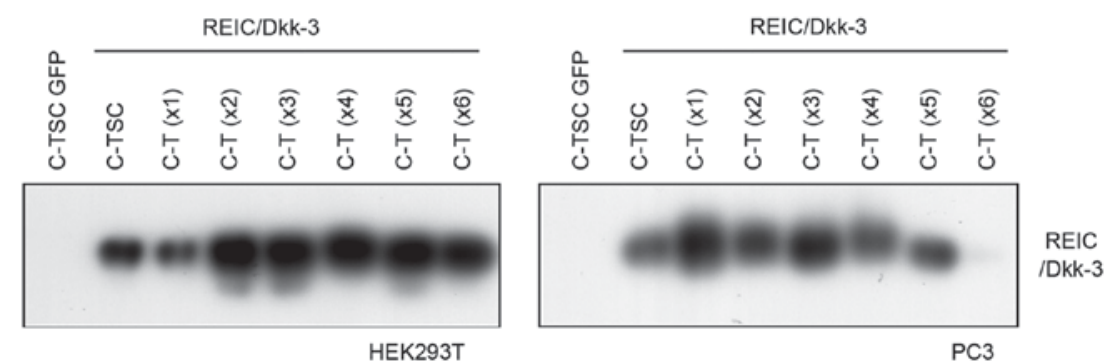

Figure 1. Schematic diagram of the improved gene expression system and assessment of its gene expression. (A) A series of indicated plasmids were constructed on the basis of the promoter-less pIDT-SMART (Km) vector. (B) Expression of REIC/Dkk-3 protein was assessed by western blotting following transfection with the indicated plasmids in HEK293T and PC3 cells. C-TSC, cassette containing cytomegalovirus-RU5' upstream, human telomerase reverse transcriptase, simian virus 40 and cytomegalovirus downstream, with a polyadenylation signal; CMV, cytomegalovirus; MCS, multi-cloning site; BGH, bovine growth hormone; polyA, polyadenylation; p, phosphorylated; hTERT, human telomerase reverse transcriptase; REIC, reduced expression in immortalized cells; Dkk-3, Dickkopf-3; GFP, green fluorescent protein C-T, cassette containing cytomegalovirus-RU5' upstream, human telomerase reverse transcriptase downstream and a polyadenylation signal.

Apoptosis assay. Apoptotic cells were analyzed as per a previously described method (9). Cells $\left(1 \times 10^{5}\right.$ cells/well) were seeded in 6-well plates and incubated for $24 \mathrm{~h}$ at $37^{\circ} \mathrm{C}$. At $48 \mathrm{~h}$ after transfection, Hoechst $33342(2 \mu \mathrm{g} / \mathrm{ml})$ was added into the culture medium and the cells were incubated for a further $30 \mathrm{~min}$. Apoptotic cells were detected using fluorescence microscopy (magnification, x400). Cell counts were manually performed from 5 fields of view for each well; the experiment was performed in triplicate.

$X$-gal staining. Cells $\left(1 \times 10^{5}\right.$ cells/well in 12 -well plates) were seeded and incubated at $37^{\circ} \mathrm{C}$ for $24 \mathrm{~h}$. At $48 \mathrm{~h}$ after infection at the indicated MOI, X-gal staining was performed with a $\beta$-gal Staining kit (cat. no. K1465-01; Thermo Fisher Scientific, Inc.), according to the manufacturer's protocol. Using optical microscopy (BZ-X710; Keyence Co., Osaka, Japan; magnification, $\mathrm{x} 100$ ), the number of infected cells were manually counted from 3 fields of view for each well. The experiment was repeated in triplicate.
Titration of adenovirus production. Adenovirus production was assessed in HEK293 cells. HEK293 donor cells $\left(2 \times 10^{5}\right.$ cells in $10-\mathrm{cm}$ dishes) were seeded and incubated for $24 \mathrm{~h}$ at $37^{\circ} \mathrm{C}$. Ad-C-TSC-REIC and Ad-C-T-REIC were infected at $2 \mathrm{MOI}$ into HEK 293 cells at $37^{\circ} \mathrm{C}$ for $48 \mathrm{~h}$. Subsequent to infection, cells were collected and ruptured with 3 freeze and thaw cycles. Purification of the adenovirus from the collected cell extracts was performed using a commercially available small column, ViraBind Adenovirus Miniprep kit (Cell Biolabs, Inc., San Diego, CA, USA), according to the manufacturer's protocol. The titer of the purified virus was determined using an Adeno-X Rapid Titer kit (Takara Biotechnology Co., Ltd.) according to the manufacturer's protocol. Using optical microscopy as previously described, infected cells were identified. Infected cell counts were performed for 20 visual fields per well at x100 magnification, to evaluate the number of plaque-forming units.

Statistical analysis. Results are expressed as the mean \pm standard deviation, unless otherwise indicated. Unpaired Student's 
A

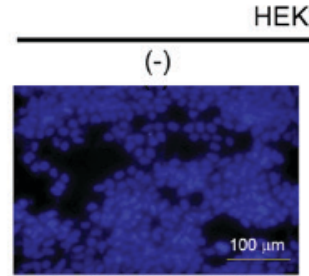

C-T (x1) REIC/Dkk-3

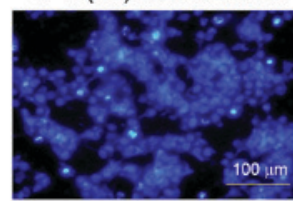

C-T (x3) REIC/Dkk-3

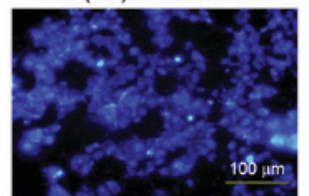

C-T (x5) REIC/Dkk-3

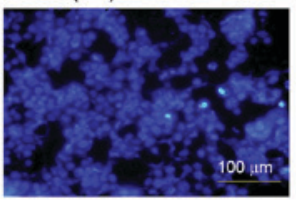

HEK293T

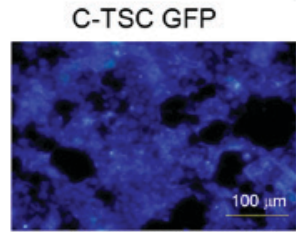

C-T (x2) REIC/Dkk-3

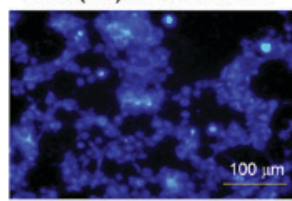

C-T (x4) REIC/Dkk-3

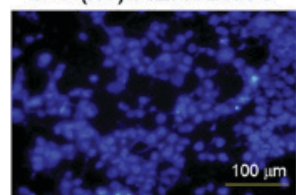

C-T (x6) REIC/Dkk-3

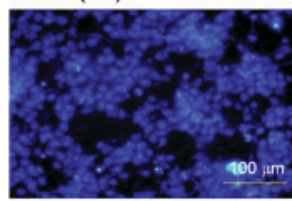

PC3

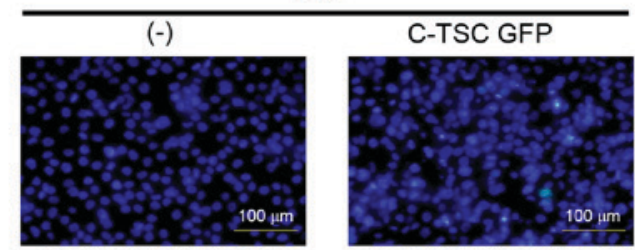

C-T (x1) REIC/Dkk-3
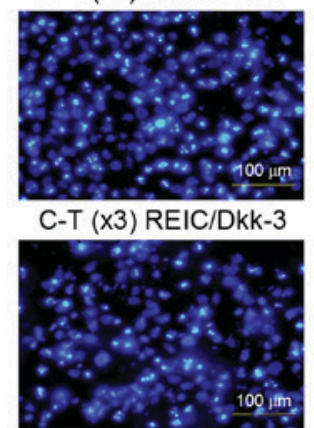

C-T (x5) REIC/Dkk-3

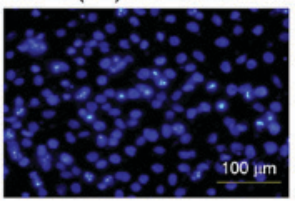

C-T (x2) REIC/Dkk-3

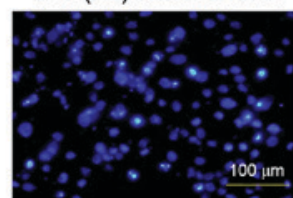

C-T (x4) REIC/Dkk-3

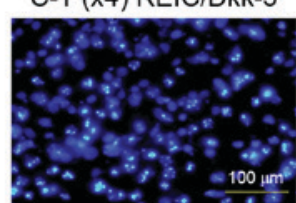

C-T (x6) REIC/Dkk-3

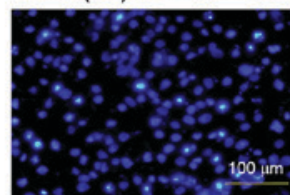

B
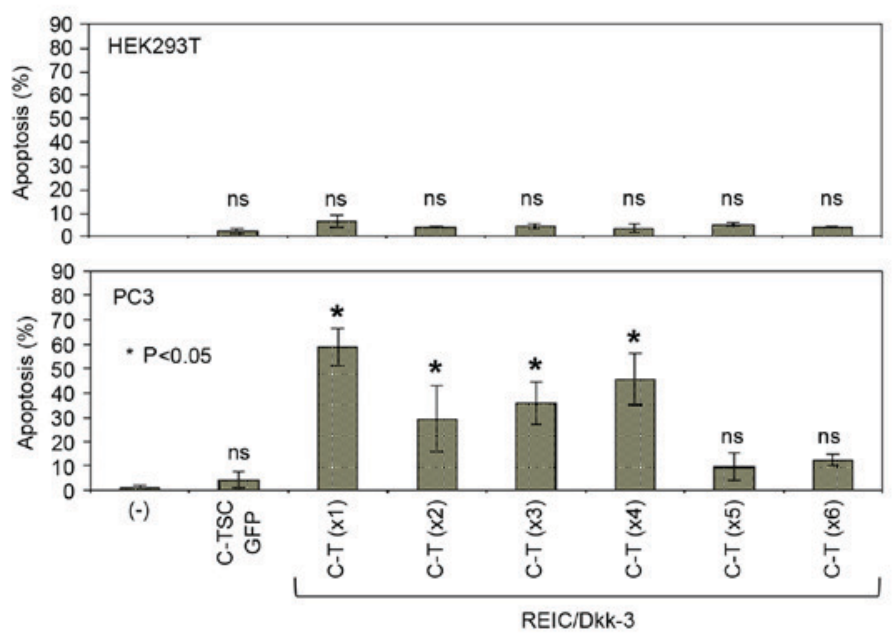

Figure 2. Assessment of newly designed plasmid vectors for their apoptosis-inducing capabilities. (A) Cells transfected with the indicated plasmids were incubated for $48 \mathrm{~h}$ and subsequently stained with Hoechst 33342. (B) Hoechst-positive cells were counted as an index of apoptotic cells. All treatments were performed in triplicate and cell counts were performed from the sum of five images from each well with fluorescence microscopy and averaged from three wells. C-TSC, cassette containing cytomegalovirus-RU5' upstream, human telomerase reverse transcriptase, simian virus 40 and cytomegalovirus downstream, with a polyadenylation signal; GFP, green fluorescent protein; C-T, cassette containing cytomegalovirus-RU5' upstream, human telomerase reverse transcriptase downstream and a polyadenylation signal; REIC, reduced expression in immortalized cells; Dkk-3, Dickkopf-3; ns, not significant.

t-tests were performed for statistical analyses using Microsoft Excel 2016 (Microsoft Corporation, Redmond, WA, USA). $\mathrm{P}<0.05$ was considered to indicate a statistically significant difference.

\section{Results}

Assessment of the plasmids for gene expression levels and induction of apoptotic cell death. In order to improve the Ad-C-TSC-REIC vector and make it more permissive in donor HEK293 cells, without losing its high expression level in cancer cells, the present study modified the 3'-TSC (phTERT-pSV40-pCMV) region in the C-TSC cassette, as the 3 '-region is known to affect gene expression level in vectors (7). To further simplify and weaken the 3'-TSC, the cancer-specific hTERT promoter (phTERT:T) was selected among the components of the 3'-TSC construct, since either pSV40 or pCMV was expected to demonstrate higher promoter activity compared with that of phTERT, based on previous studies $(7,8)$. The present study first aimed to make a series of improved constructs using phTERT as the basis of the C-TSC vector structure. To determine the optimal number of phTERT at the 3'-side of the cDNA, six constructs were prepared, termed C-T ( $\mathrm{x} 1$ to $\mathrm{x} 6)$, where phTERT was serially repeated up to six (Fig. 1A). Following insertion of REIC/Dkk-3 into the multi-cloning site of the indicated plasmid constructs, 

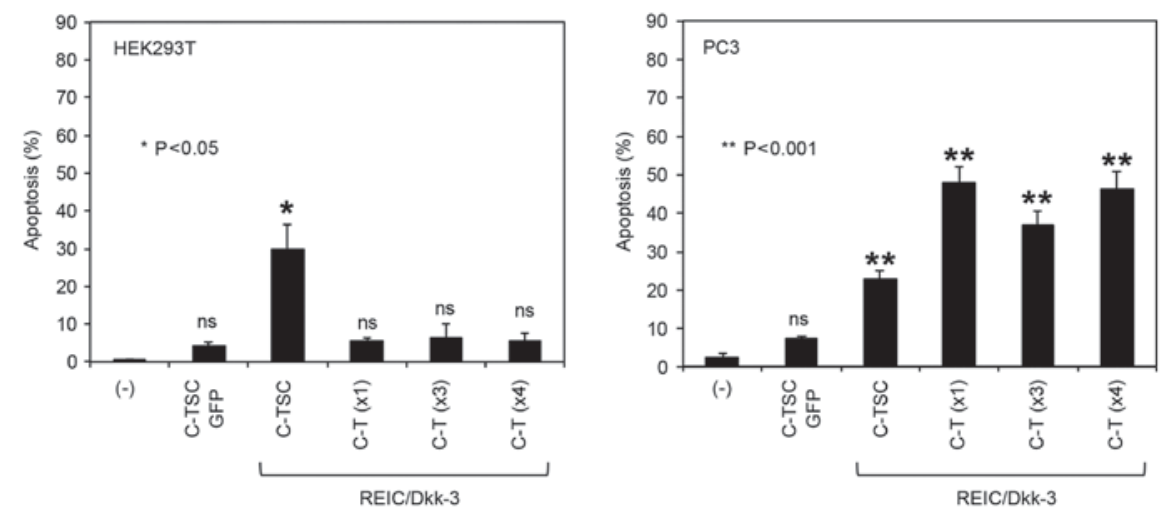

Figure 3. Repeat assessment of the apoptosis-inducing capabilities of the novel plasmid vectors, in comparison with the C-TSC plasmid. Following 48-h transfection of cells with the indicated plasmids, the cells were stained with Hoechst 33342 and the number of apoptotic cells were counted. All treatments were performed in triplicate and cell counts were performed from the sum of five images from each well and averaged from three wells. C-TSC, cassette containing cytomegalovirus-RU5' upstream, human telomerase reverse transcriptase, simian virus 40 and cytomegalovirus downstream, with a polyadenylation signal; GFP, green fluorescent protein; C-T, cassette containing cytomegalovirus-RU5' upstream, human telomerase reverse transcriptase downstream and a polyadenylation signal; REIC, reduced expression in immortalized cells; Dkk-3, Dickkopf-3; ns, not significant.
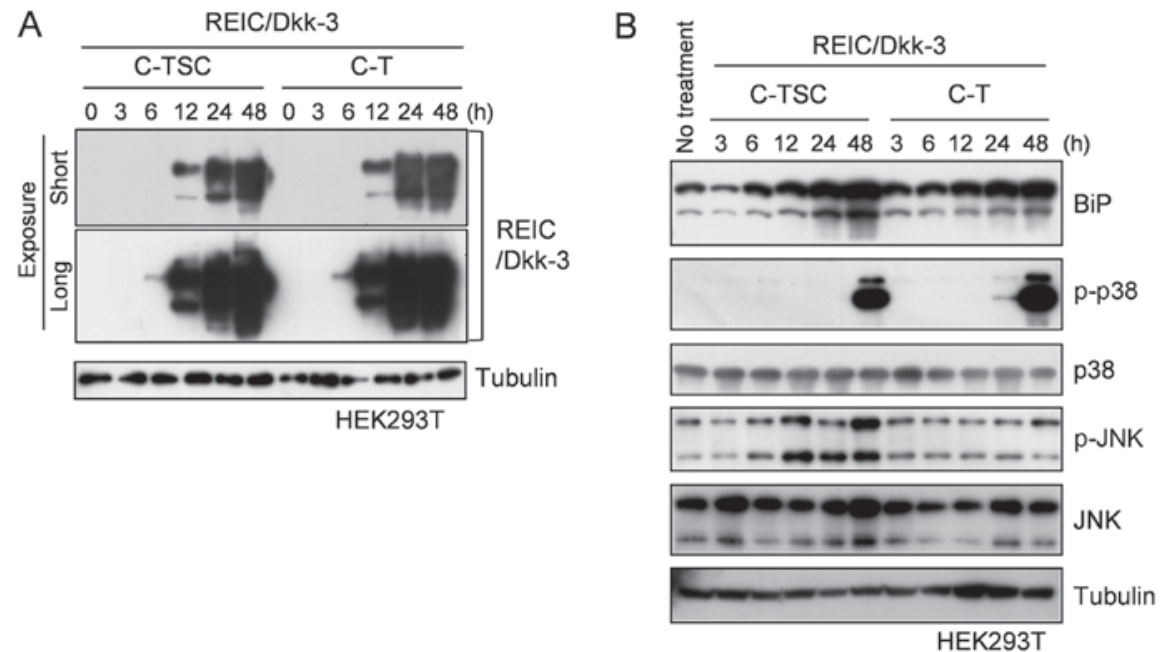

Figure 4. Time course analysis of REIC/Dkk-3 expression levels and associated intracellular signaling. (A) Time course evaluation of REIC/Dkk-3 expression levels from REIC/Dkk-3 cDNA-carrying C-TSC and C-T plasmids was performed in HEK293T cells by western blotting. (B) Protein expression levels of the endoplasmic reticulum stress marker BiP and activation levels of $\mathrm{p} 38$ and JNK were analyzed by western blotting. REIC, reduced expression in immortalized cells; Dkk-3, Dickkopf-3; C-TSC, cassette containing cytomegalovirus-RU5' upstream, human telomerase reverse transcriptase, simian virus 40 and cytomegalovirus downstream, with a polyadenylation signal; C-T, cassette containing cytomegalovirus-RU5' upstream, human telomerase reverse transcriptase downstream and a polyadenylation signal; BiP, binding immunoglobulin protein; p, phosphorylated; JNK, c-Jun N-terminal kinase.

temporal transfection experiments were performed using the newly constructed C-T vectors and the C-TSC vector, and the performance of each of the vectors for expression of the REIC/Dkk-3 gene was assessed. Western blotting revealed that the expression level of C-T (x1) in HEK293T cells was comparable to or slightly lower compared with that of C-TSC, whereas the remaining C-T constructs (x2-x6) demonstrated further increases in expression to almost the same levels. Conversely, in prostate cancer PC3 cells, C-T (x1) exhibited the highest expression level of REIC/Dkk-3 in the tested constructs (Fig. 1B). Of note, C-T (x6) did not induce any appreciable level of REIC/Dkk-3 expression in PC3 cells.

Under the same experimental conditions, the present study then assessed whether apoptotic cell death was initiated using the newly constructed plasmids, since overexpression of REIC/Dkk-3 is able to induce apoptosis in a various types of cancer cell (3-6). Hoechst staining is a conventional and reliable method for detecting apoptotic cell death in which cells express bright fluorescence, particularly in the apoptosis-mediated condensed nuclei, and therefore this method was used to assess effects of the indicated constructs (Fig. 2A). Combined with the results of quantification (Fig. 2B), it was revealed that despite the higher expression levels of REIC/Dkk-3 derived from C-T (x1-6) plasmids, apoptosis was hardly detected in HEK293 cells, whereas a high rate of apoptosis was observed in PC3 cells transfected with C-T (x1-4), but not in those transfected with C-T (x5 and $\mathrm{x} 6$ ). The greatest effect was induced by the C-T (x1) construct. Repeated experiments using the C-T constructs together with the C-TSC construct also demonstrated that, although REIC/Dkk-3 produced by the $\mathrm{C}$-TSC construct induced a high rate of apoptotic cell death in HEK293T and PC3 cells, the C-T (x1, x3 and x4) constructs selectively induced high rates of apoptotic cell death in PC3 cells but not in HEK293T cells (Fig. 3). As a result, the C-T 
A

A
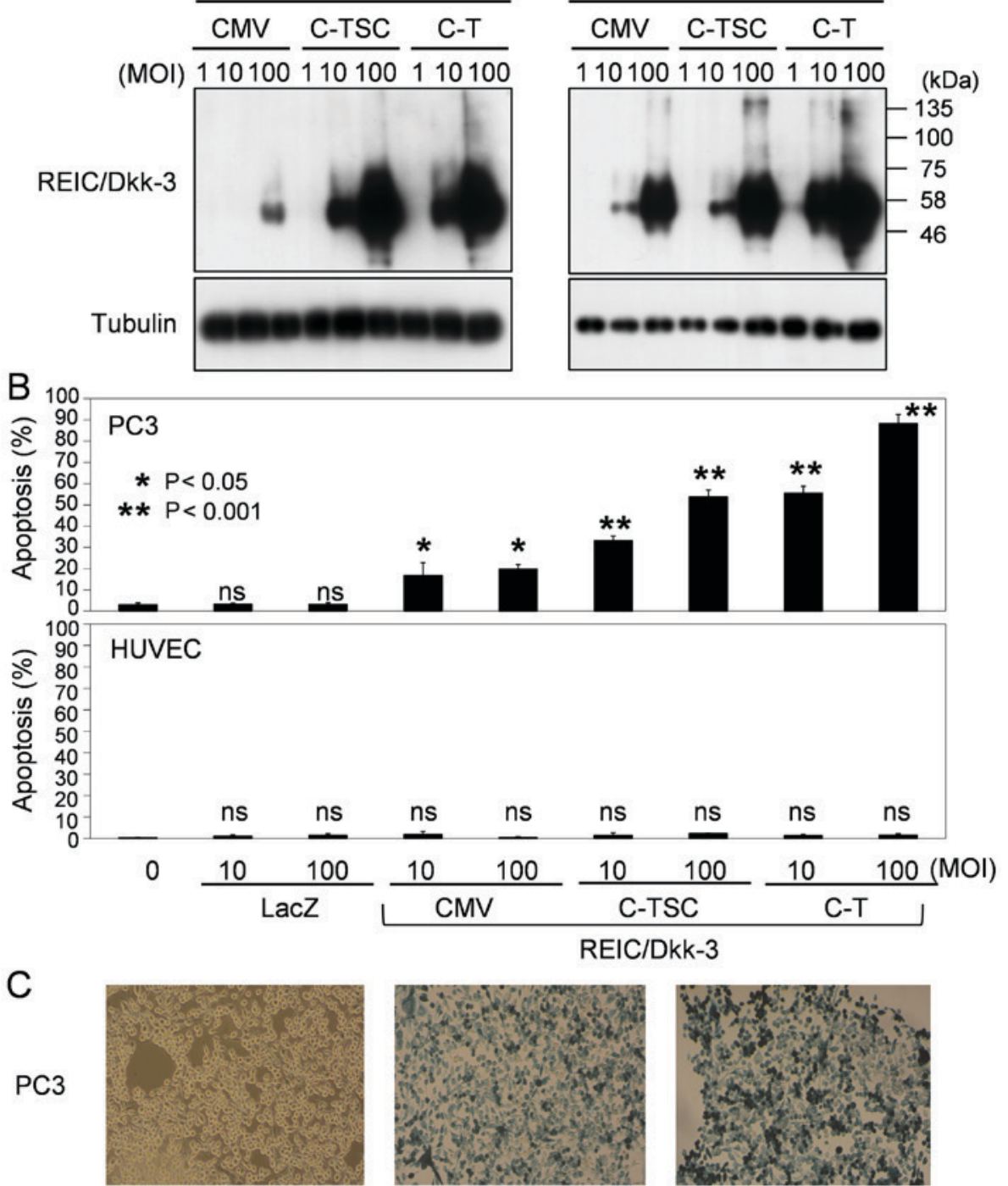

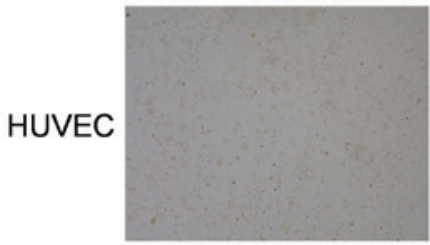

$(-)$
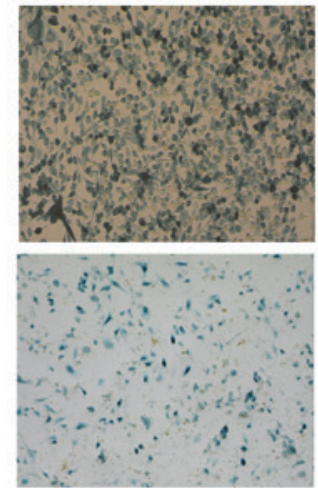

LacZ $10 \mathrm{MOI}$
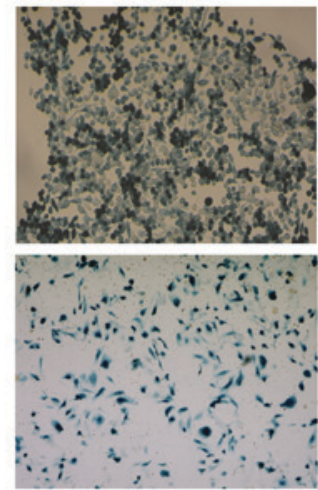

LacZ $100 \mathrm{MOI}$

Figure 5. Adaptation of the C-T cassette to the conventional Ad-REIC and evaluation of the adapted vector, Ad-C-T-REIC, for gene expression and induction of apoptosis. (A) Expression levels of REIC/Dkk-3 from the indicated adenovirus vectors was determined in a human prostate cancer cell line (PC-3) and normal HUVECs. Tubulin was used as a control for loaded amounts of protein. (B) Induction of apoptosis in PC 3 and HUVEC cells by the indicated adenovirus vectors carrying REIC/Dkk-3. (C) Assessment of infection efficiency in PC3 and HUVEC cells by infection with the conventional Ad-LacZ at MOIs of 10 and 100. Expression of the LacZ gene was assessed by X-gal staining. C-T, cassette containing cytomegalovirus-RU5' upstream, human telomerase reverse transcriptase downstream and a polyadenylation signal; Ad, adenovirus; REIC, reduced expression in immortalized cells; Dkk-3, Dickkopf-3; HUVECs, human umbilical vein endothelial cells; LacZ, $\beta$-galactosidase; MOI, multiplicity of infection; C-TSC, cassette containing cytomegalovirus-RU5' upstream, human telomerase reverse transcriptase, simian virus 40 and cytomegalovirus downstream, with a polyadenylation signal; CMV, cytomegalovirus; ns, not significant.

(x1) construct was selected for further analysis, and termed 'C-T' due to its simpler vector structure.

Transfection with $C$-TSC and $C$-T plasmids results in similar levels of REIC/Dkk-3 signaling, and BiP protein was elevated in cells transfected with C-TSC only. The present study next investigated why the rates of apoptosis in HEK293T cells and PC3 cells were different. One potential reason may be that ER stress-associated apoptosis is induced by REIC/Dkk-3 overexpression, which induces JNK activation, which in turn is linked to cancer-specific cell death (3-6). In general, ER stress is affected by the folding speed of a newly synthesized protein; therefore, the present study examined the speed of expression of the REIC/Dkk-3 protein in HEK293T cells transfected with C-TSC and C-T plasmids. However, no difference was observed in REIC/Dkk-3 protein expression levels across a time course experiment in cells transfected with the two vectors (Fig. 4A). The elevation of the ER stress marker 
BiP protein, which was linked to the activation of JNK in a parallel manner in C-TSC-transfected cells, was confirmed (Fig. 4B). Notably, this did not occur in C-T-transfected cells, which suggested the presence of an unknown mechanism, or mechanisms, independent of simple REIC/Dkk-3 protein expression triggering ER stress, followed by JNK-mediated cell death.

Adaptation of the C-T cassette to the conventional Ad-REIC. To further validate the utility of the C-T construct, the $\mathrm{C}-\mathrm{T}$ composition was adapted to an adenovirus vector and an improved Ad-C-T-REIC adenovirus vector was developed. The REIC/Dkk-3 expression levels were compared by western blotting in cells transfected with the newly produced Ad-C-T-REIC and the common CMV and C-TSC cassette-driven adenovirus vectors (Ad-CMV-REIC and Ad-C-TSC-REIC; Fig. 5). In comparison with the common Ad-CMV-REIC, Ad-C-T-REIC significantly enhanced the expression of REIC/Dkk-3 in PC3 cancer cells and normal HUVEC cells. These expression levels were comparable to those of Ad-C-TSC-REIC in PC3 cells, whereas the expression levels tended to further increase in HUVEC cells (Fig. 5A). To investigate the therapeutic utility of Ad-C-T-REIC in human cancer cells, the present study then assessed the in vitro apoptotic effects of the vector under the same conditions as those presented in Fig. 5A. Notably, the Ad-C-T-REIC exhibited markedly higher induction of apoptosis in PC3 cells with MOI 100 (Fig. 5B). Similar results were also obtained in an aggressive human glioblastoma cell line, T98 (data not shown). The present study confirmed that there was no notable difference in the infectious efficiency of the adenovirus vector between PC3 and HUVEC cells (Fig. 5C). Finally, it was demonstrated that viral production was 2 -fold higher with Ad-C-T-REIC compared with Ad-C-TSC-REIC (Fig. 6; Table I). Taken together, the results indicated that the improved $\mathrm{C}-\mathrm{T}$ cassette enhanced gene expression to a level comparable to the C-TSC cassette, and increased adenoviral production in HEK293 cells. Hence, the Ad-C-T-REIC vector has potential to become a useful tool for cancer gene therapy.

\section{Discussion}

The previously developed adenovirus vector carrying REIC/Dkk-3, Ad-C-TSC-REIC, is expected to become an innovative cancer therapeutic tool due to its notably powerful gene expression $(7,8)$. However, the significantly reduced rate of adenoviral production by HEK293 cells due to the higher expression level of REIC/Dkk-3, or for an unknown reason, with the Ad-C-TSC-REIC vector remains an issue that requires consideration. Therefore, the present study aimed to establish a method for resolving this issue. The present study attempted to identify a solution via modification of the original Ad-C-TSC-REIC construct. The 3'-TSC region was replaced with a single hTERT promoter, resulting in the creation of the Ad-C-T-REIC vector. The improved vector demonstrated significantly higher expression of REIC/Dkk-3, which was comparable to that of the Ad-C-TSC-REIC vector, but with attenuation of apoptotic cell death in donor HEK293 cells. This resulted in a two-fold elevation of viral production compared
Table I. PFU of the stained HEK293 cells.

\begin{tabular}{lcc}
\hline Parameter & Ad-C-TSC-REIC & Ad-C-T-REIC \\
\hline Hexon-positive cells, $\mathrm{n}$ & $35.8 \pm 6.9$ & $62.2 \pm 14.0$ \\
Titer, PFU/ml & $8.62 \pm 1.7 \times 10^{9}$ & $15 \pm 3.4 \times 10^{9}$ \\
\hline
\end{tabular}

Data are presented as the mean \pm standard deviation $(n=20)$. PFU, plaque forming units; Ad, adenovirus; C-TSC, cassette containing cytomegalovirus-RU5' upstream, human telomerase reverse transcriptase, simian virus 40 and cytomegalovirus downstream, with a polyadenylation signal; REIC, reduced expression in immortalized cells; C-T, cassette containing cytomegalovirus-RU5' upstream, human telomerase reverse transcriptase downstream and a polyadenylation signal.

\section{HEK293}

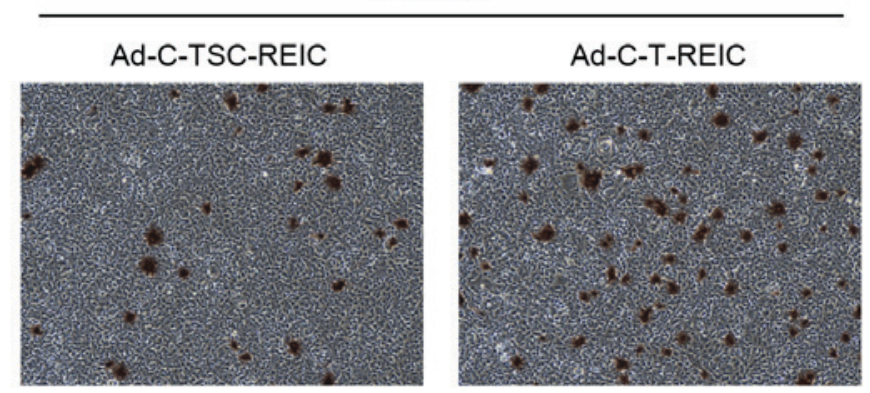

Figure 6. Evaluation of viral production using Ad-C-TSC-REIC and Ad-C-T-REIC. HEK293 cells were infected with Ad-C-TSC-REIC and Ad-C-T-REIC. Cells were lysed and the virus was purified from the cell extracts. The titer of the purified virus was determined using an Adeno-X Rapid Titer kit; using optical microscopy, infected cells were counted from 20 images per each well. Magnification, x100. Ad, adenovirus; C-TSC, cassette containing cytomegalovirus-RU5' upstream, human telomerase reverse transcriptase, simian virus 40 and cytomegalovirus downstream, with a polyadenylation signal; REIC, reduced expression in immortalized cells; C-T, cassette containing cytomegalovirus-RU5' upstream, human telomerase reverse transcriptase downstream and a polyadenylation signal.

with that observed in the case of Ad-C-TSC-REIC, suggesting that the improved Ad-C-T-REIC vector may be valuable for application to cancer gene therapy.

The hTERT promoter is generally used to provide the cargo gene within a certain vector for cancer-specific expression; however, its insufficient gene expression activity remains a serious issue. Therefore, it has been reported that the combination of the hTERT promoter with multiple enhancer elements from other promoters may significantly enhance the gene expression in comparison to that of the control plasmid vectors (10). Notably, in the present study it was revealed that positioning the hTERT promoter at the 3 '-side of the cDNA alone was sufficient to produce a significant effect on gene expression level without the need for a serial combination with other promoters or enhancers. Nevertheless, the 3'-side of the hTERT promoter may not exhibit cancer specificity, since strong expression of REIC/Dkk-3 was observed in normal human HUVEC cells, which may in part be due to the 5'-side of the CMV promoter.

At present, it remains difficult to explain the difference in apoptosis that was observed between the C-TSC and C-T systems in HEK293T cells. As indicated by the results of the 
present study, this was not only dependent on the REIC/Dkk-3 expression level and speed. Our previous study demonstrated that overexpression of REIC/Dkk-3 induced cancer-specific apoptosis by triggering certain ER stress markers and subsequent JNK activation (3-6). Notably, ER stress and JNK activation were mitigated by the $\mathrm{C}-\mathrm{T}$ vector compared with transfection with the C-TSC vector. In this respect, a reasonable explanation remains to be elucidated, as the molecular mechanism underlying gene expression levels in the newly developed vector require detailed clarification (7). Further, more extensive studies are required to clarify the perplexity of the expression principle and intracellular events that may have been triggered by numerous materials in the present study (for example, vector-derived untranslated RNAs that may function as microRNAs or others), including the REIC/Dkk-3 protein produced by the novel system. Further studies in this area will ultimately result in an improved understanding of the vector principles required to produce higher and more specific levels of gene expression dependent on cell types, and contribute to the development of therapeutic approaches for a number of subtypes of cancer.

\section{Acknowledgements}

The present study was supported by the Japanese Society for the Promotion of Science (KAKENHI; grant nos. JP26290039 and JP15K14382). The authors declare the following conflicts of interest, Okayama University is applying for patents for the new vector systems. Dr Masakiyo Sakaguchi, Dr Masami Watanabe, Dr Nam-ho Huh and Dr Hiromi Kumon are the designated inventors for the patents. Okayama University and Momotaro-Gene, Inc. (Okayama, Japan) are working together for the development of the Ad-REIC agent; Momotaro-Gene, Inc. holds the patents for the Ad-REIC agent. Dr Masakiyo Sakaguchi, Dr Masami Watanabe, Dr Nam-ho Huh, Dr Yasutomo Nasu and Dr Hiromi Kumon own stock in Momotaro-Gene, Inc.

\section{References}

1. Tsuji T, Miyazaki M, Sakaguchi M, Inoue Y and Namba M: A REIC gene shows down-regulation in human immortalized cells and human tumor-derived cell lines. Biochem Biophys Res Commun 268: 20-24, 2000.

2. Tsuji T, Nozaki I, Miyazaki M, Sakaguchi M, Pu H, Hamazaki Y, Iijima O and Namba M: Antiproliferative activity of REIC/Dkk-3 and its significant down-regulation in non-small-cell lung carcinomas. Biochem Biophys Res Commun 289: 257-263, 2001.

3. Abarzua F, Sakaguchi M, Takaishi M, Nasu Y, Kurose K, Ebara S, Miyazaki M, Namba M, Kumon $\mathrm{H}$ and Huh NH: Adenovirus-mediated overexpression of REIC/Dkk-3 selectively induces apoptosis in human prostate cancer cells through activation of c-Jun-NH2-kinase. Cancer Res 65: 9617-9622, 2005.

4. Kashiwakura Y, Ochiai K, Watanabe M, Abarzua F, Sakaguchi M, Takaoka M, Tanimoto R, Nasu Y, Huh NH and Kumon H: Down-regulation of inhibition of differentiation-1 via activation of activating transcription factor 3 and Smad regulates REIC/Dickkopf-3-induced apoptosis. Cancer Res 68: 8333-8341, 2008.

5. Sakaguchi M, Kataoka K, Abarzua F, Tanimoto R, Watanabe M, Murata H, Than SS, Kurose K, Kashiwakura Y, Ochiai K, et al: Overexpression of REIC/Dkk-3 in normal fibroblasts suppresses tumor growth via induction of interleukin-7. J Biol Chem 284: 14236-14244, 2009.

6. Kawasaki K, Watanabe M, Sakaguchi M, Ogasawara Y, Ochiai K, Nasu Y, Doihara H, Kashiwakura Y, Huh NH, Kumon H and Date H: REIC/Dkk-3 overexpression downregulates P-glycoprotein in multidrug-resistant MCF7/ADR cells and induces apoptosis in breast cancer. Cancer Gene Ther 16: 65-72, 2009.

7. Sakaguchi M, Watanabe M, Kinoshita R, Kaku H, Ueki H, Futami J, Murata H, Inoue Y, Li SA, Huang P, et al: Dramatic increase in expression of a transgene by insertion of promoters downstream of the cargo gene. Mol Biotechnol 56: 621-630, 2014.

8. Watanabe M, Sakaguchi M, Kinoshita R, Kaku H, Ariyoshi Y, Ueki H, Tanimoto R, Ebara S, Ochiai K, Futami J, et al: A novel gene expression system strongly enhances the anticancer effects of a REIC/Dkk-3-encoding adenoviral vector. Oncol Rep 31: 1089-1095, 2014.

9. Abarzua F, Kashiwakura Y, Takaoka M, Watanabe M, Ochiai K, Sakaguchi M, Iwawaki T, Tanimoto R, Nasu Y, Huh NH and Kumon $\mathrm{H}$ : An N-terminal 78 amino acid truncation of REIC/Dkk-3 effectively induces apoptosis. Biochem Biophys Res Commun 375: 614-618, 2008.

10. Kim SJ, Lee HS, Shin JH, Kim CG, Jeong S, Park K, Choe H and Lee H: Preferentially enhanced gene expression from a synthetic human telomerase reverse transcriptase promoter in human cancer cells. Oncol Rep 16: 975-979, 2006. 